\title{
Conditional statistics of electron transport in interacting nanoscale conductors
}

\author{
EUGENE V. SUKHORUKOV ${ }^{1 *}$, ANDREW N. JORDAN² ${ }^{2}$ SIMON GUSTAVSSON ${ }^{3}$, RENAUD LETURCQ ${ }^{3}$, \\ THOMAS IHN ${ }^{3}$ AND KLAUS ENSSLIN ${ }^{3}$
}

\author{
${ }^{1}$ Département de Physique Théorique, Université de Genève, CH-1211 Genève 4, Switzerland \\ ${ }^{2}$ Department of Physics and Astronomy, University of Rochester, Rochester, New York 14627, USA \\ ${ }^{3}$ Solid State Physics Laboratory, ETH Zurich, CH-8093 Zurich, Switzerland \\ *e-mail: eugene.sukhorukov@physics.unige.ch
}

There is an intimate connection between the acquisition of information and how this information changes the remaining uncertainty in the system. This trade-off between information and uncertainty plays a central role in the context of detection. Recent advances in the ability to make accurate, on-chip measurements of individual-electron current through a quantum $\operatorname{dot}^{1-8}$ (QD) have been enabled by exploiting the sensitivity of a second current, passing through a nearby quantum point contact (QPC), to the fluctuating charge on the $\mathrm{QD}^{4-8}$. An important characteristic of $\mathrm{QPC}$ detectors is their minimal influence on the systems they probe. Here we show that even the operation of an effectively non-invasive QPC detector can statistically alter the system's behaviour. By observing a particular QPC current, the statistical distribution of the QD conditional current undergoes a substantial change in comparison to that expected for unconditional shot noise ${ }^{9}$. These results are in almost perfect agreement with a theoretical model we develop to predict the joint current probability distribution and conditional transport statistics of interacting nanoscale systems.

Noise is generally due to randomness, which can be classical or quantum in nature. Telegraph noise, where there is random switching between two stable states ${ }^{10}$, originates from such diverse phenomena as thermal activation of an unstable impurity ${ }^{11-13}$, non-equilibrium activation of a bistable system ${ }^{14-17}$, switching of magnetic domain orientation ${ }^{18-20}$, or a reversible chemical reaction in a biological ion channel ${ }^{21}$.

In nanoscale conductors, where charge motion is quantum coherent over distances comparable to the system size, shot noise and telegraph noise have recently been shown to be two sides of the same coin ${ }^{6,7,22,23}$. A quantum dot (QD) is sufficiently small that it is effectively zero dimensional, and behaves as an artificial atom, holding a small number of electrons. Figure 1a shows the sample used in the experiment reported here. The QD is marked by the dotted circle $^{24}$. An extra electron can tunnel into the QD from the source lead (S), stay in the QD for a random amount of time and then tunnel out into the drain lead (D) if the applied voltage bias exceeds the temperature. This singleelectron transport produces a fluctuating electrical current. In order to detect the statistical properties of this current, a sensitive electrometer with a bandwidth much higher than the tunnelling rates is required. The electrometer is a nearby quantum point contact (QPC) that is capacitively coupled to the QD via the Coulomb interaction. The voltage-biased QPC detector transports many electrons through a narrow constriction in the surrounding two-dimensional electron gas (represented with an arrow). The resistance of the QPC is susceptible to changes in the surrounding electrostatic environment, and can therefore be used to sense the presence (or absence) of an extra electron on the $\mathrm{QD}^{4}$. When the extra electron tunnels into or out of the QD, the current $I$ flowing through the QPC switches between two different values (see Fig. 1c). Therefore, the shot noise of the QD current $J$ (randomness in the number of switches in a given time interval) is intimately linked with telegraph noise in the QPC current $I$ (randomness of duration time in each current value).

In the following, we develop a theoretical model for this experiment. Ideal switching of the measured detector signal between two noiseless values $I_{1,2}$ is identified with the two current levels experienced by the QPC. When an electron enters the QD the current switches from $I_{1}$ to $I_{2}$, and when the electron leaves the QD the reverse switch happens. The number of 'down' or 'up' switches $M$ in a given time trace of duration $t$ is identified with the number of different transport electrons that occupy the QD in that time interval, naturally defining a QD current variable $J=M / t$ (we set $e=1$ to count in single-electron charge units). The analogous number of electrons $N$ passed by the QPC in this same time interval defines the QPC current variable $I=N / t$. The assumption of noiseless current levels implies that $I_{1} \leq I(t) \leq I_{2}$, and the unidirectional nature of the QD transport implies that $0 \leq J(t)<\infty$ (see the Supplementary Information for justification). Stochastic, statistically independent quantum tunnelling into and out of the QD is described with rates $\Gamma_{1,2}$ (ref. 22). For later convenience, we define the average and difference variables $I_{0}=\left(I_{1}+I_{2}\right) / 2, \Gamma_{0}=\left(\Gamma_{1}+\Gamma_{2}\right) / 2$ and $\Delta I=\left(I_{2}-I_{1}\right) / 2, \Delta \Gamma=\left(\Gamma_{2}-\Gamma_{1}\right) / 2$. This model is capable of describing a host of phenomena in many fields of science.

Taken alone, each side of the random process may be characterized mathematically with the probability distributions $P(I, t)$ and $P(J, t)$ of finding a given number of electrons transmitted in a given time, or equivalently all current cumulants $\left\langle\left\langle I^{n}\right\rangle\right.$ and $\left\langle\left\langle J^{m}\right\rangle\right\rangle$ (see the Supplementary Information for a discussion of these statistical quantities). This catalogue of 

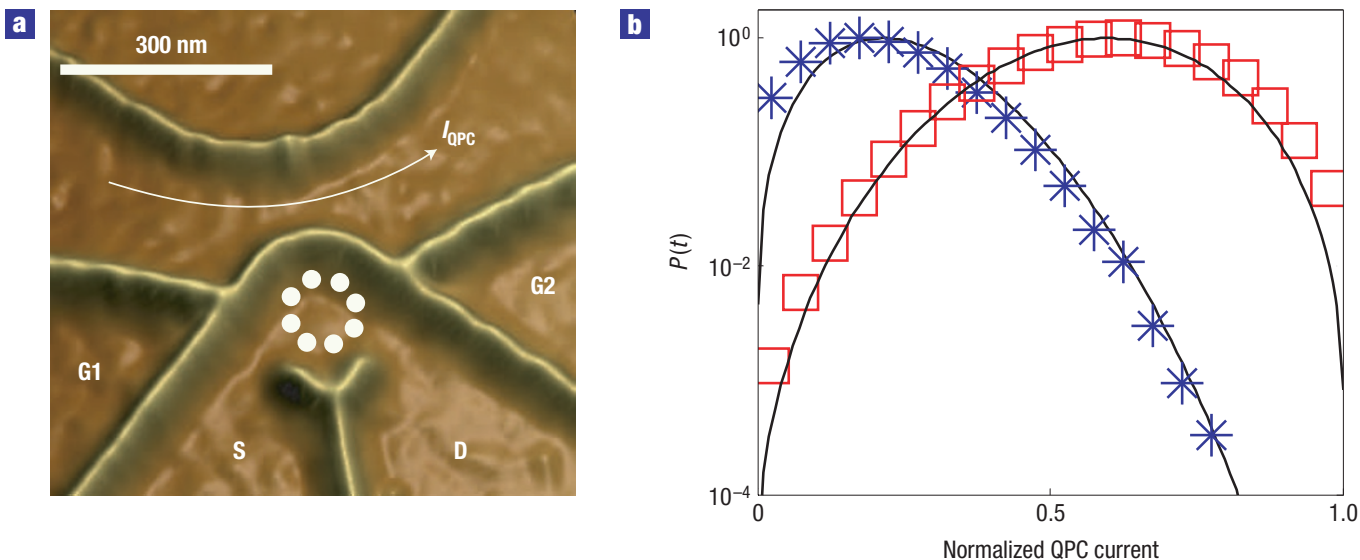

G

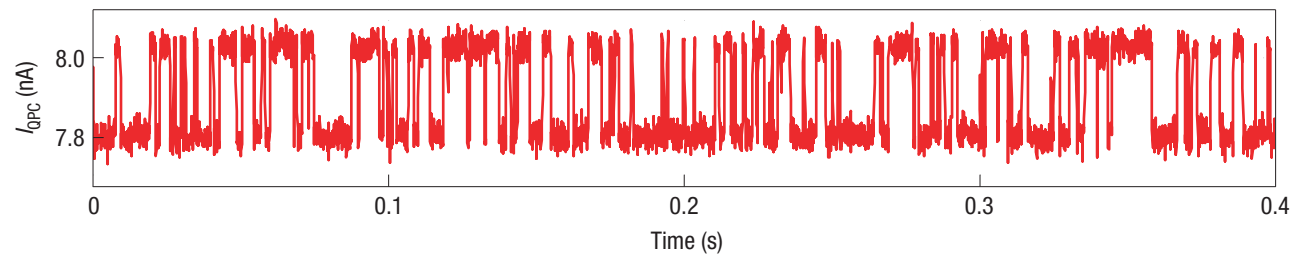

Figure 1 Nanodevice and current fluctuations. a, Quantum dot-quantum point contact structure fabricated using scanning probe lithography ${ }^{24}$ on a GaAs $/ \mathrm{Al}_{0.3} \mathrm{Ga}_{0.7} \mathrm{As}_{\mathrm{S}}$ heterostructure containing a two-dimensional electron gas $34 \mathrm{~nm}$ below the surface. Ballistic electron flow through the QPC is indicated with an arrow, and the quantum dot is indicated with a dotted circle. Individual-electron current is induced by applying a voltage bias between source (S) and drain (D). b, Probability distribution of charge transmitted through the QPC, plotted on a logarithmic scale. Data set A is represented by blue asterisks and data set B is represented by open red squares. The solid lines are the theoretical prediction of a universal ellipse for both configurations, with no fitting parameter. Slight deviations from the ellipse at the ends of the interval are due to small-amplitude noise fluctuations in the individual current levels ${ }^{28}$. c, The flow of single-electron current is deduced by the presence of switching in the QPC current, which acts as an on-chip electrometer.

cumulants gives a unique signature of the particular electronic conductor, and is also known as full counting statistics ${ }^{25}$. For example, the first two cumulants are the average current $\langle I\rangle$ and the shot noise power $\left\langle\left\langle I^{2}\right\rangle\right\rangle=\int \mathrm{d} t\langle\delta I(t) \delta I(0)\rangle$, where $\delta I=I(t)-\langle I\rangle$. For the QPC and QD, the average currents $\langle I\rangle$ and $\langle J\rangle$ and shot noise powers $\left\langle\left\langle I^{2}\right\rangle\right\rangle$ and $\left\langle\left\langle J^{2}\right\rangle\right\rangle$ are given respectively by

$$
\begin{gathered}
\langle I\rangle=\left(I_{1} \Gamma_{2}+I_{2} \Gamma_{1}\right) /\left(2 \Gamma_{0}\right), \quad\left\langle\left\langle I^{2}\right\rangle\right\rangle=(\Delta I)^{2} \Gamma_{1} \Gamma_{2} / \Gamma_{0}^{3}, \\
\langle J\rangle=\Gamma_{1} \Gamma_{2} /\left(2 \Gamma_{0}\right), \quad\left\langle\left\langle J^{2}\right\rangle\right\rangle=\langle J\rangle\left(\Gamma_{1}^{2}+\Gamma_{2}^{2}\right) /\left(4 \Gamma_{0}^{2}\right) .
\end{gathered}
$$

In the limit of small switching rates $\Gamma_{1,2} \rightarrow 0$ the current and noise of the QD vanish, whereas the noise of the QPC actually diverges.

However, a simple specification of the counting statistics of each conductor individually misses the important fact that the two conductors are strongly correlated by the Coulomb interaction between them. The simplest measure of the correlation between the systems is the cross-correlation $\left\langle\langle I J\rangle=\int \mathrm{d} t\langle\delta I(t) \delta J(0)\rangle\right.$, given by

$$
\langle\langle J\rangle\rangle=(\Delta I \Delta \Gamma) \Gamma_{1} \Gamma_{2} / \Gamma_{0}^{2} .
$$

This correlator is approximately constant under scaling of the switching rates, compromising between the behaviour of the two noises individually (1). Result (2) implies that the two currents may be either positively or negatively correlated, depending on the sign of $\Delta \Gamma \Delta I$. This effect has a simple physical interpretation: taking $\Delta I>0$, if $\Gamma_{2}>\Gamma_{1}$, then the system typically spends more time in state 1 than in state 2 . The current $J$ is increased by adding another 'up' and 'down' switch to the current trace. This new event typically divides a long time interval spent in $I_{1}$ into two segments, subtracting a short interval from $I_{1}$ and adding it to $I_{2}$, thereby increasing the current $I$. The same argument applied with $\Gamma_{2}<\Gamma_{1}$ leads to a decrease in $I$ given an increase in $J$, explaining the sign of the cross-correlation function.

In order to specify the statistical correlation between the conductors, we introduce the joint counting statistics of both conductors. More specifically, the correlations may be quantified by the joint probability distribution $P(I, J, t)$ of finding current $I$ and current $J$ in a time $t$ (equivalently, all cross-cumulants $\left\langle\left\langle I^{n} J^{m}\right\rangle\right.$ ), or may also be specified by the conditional distribution functions $P(I \mid J)$ or $P(J \mid I)$, the probability of observing one current given an observation of the other. These distributions are all related to one another by $P(I, J)=P(I \mid J) P(J)=P(J \mid I) P(I)$, where the last equality is an expression of Bayes's theorem. The conditional distributions (and their associated conditional cumulants) play a key role in the informational approach to detection ${ }^{26}$.

From the model described above, the complete characterization of the system/detector fluctuation statistics may be obtained from conditional master equation formalism. The statistical cumulants of the current fluctuations are given with the help of a generating function $H(\lambda, \chi)$, such that the cross-cumulants are given simply by taking derivatives, $\left\langle\left\langle I^{n} J^{m}\right\rangle=\partial_{\lambda}^{n} \partial_{\chi}^{m} H(0,0)\right.$. From the mathematical derivation given in the Supplementary Information, we find that the joint generating function is given by

$$
H(\lambda, \chi)=\lambda I_{0}-\Gamma_{0}+\sqrt{(\lambda \Delta I-\Delta \Gamma)^{2}+\Gamma_{1} \Gamma_{2} \exp \chi},
$$



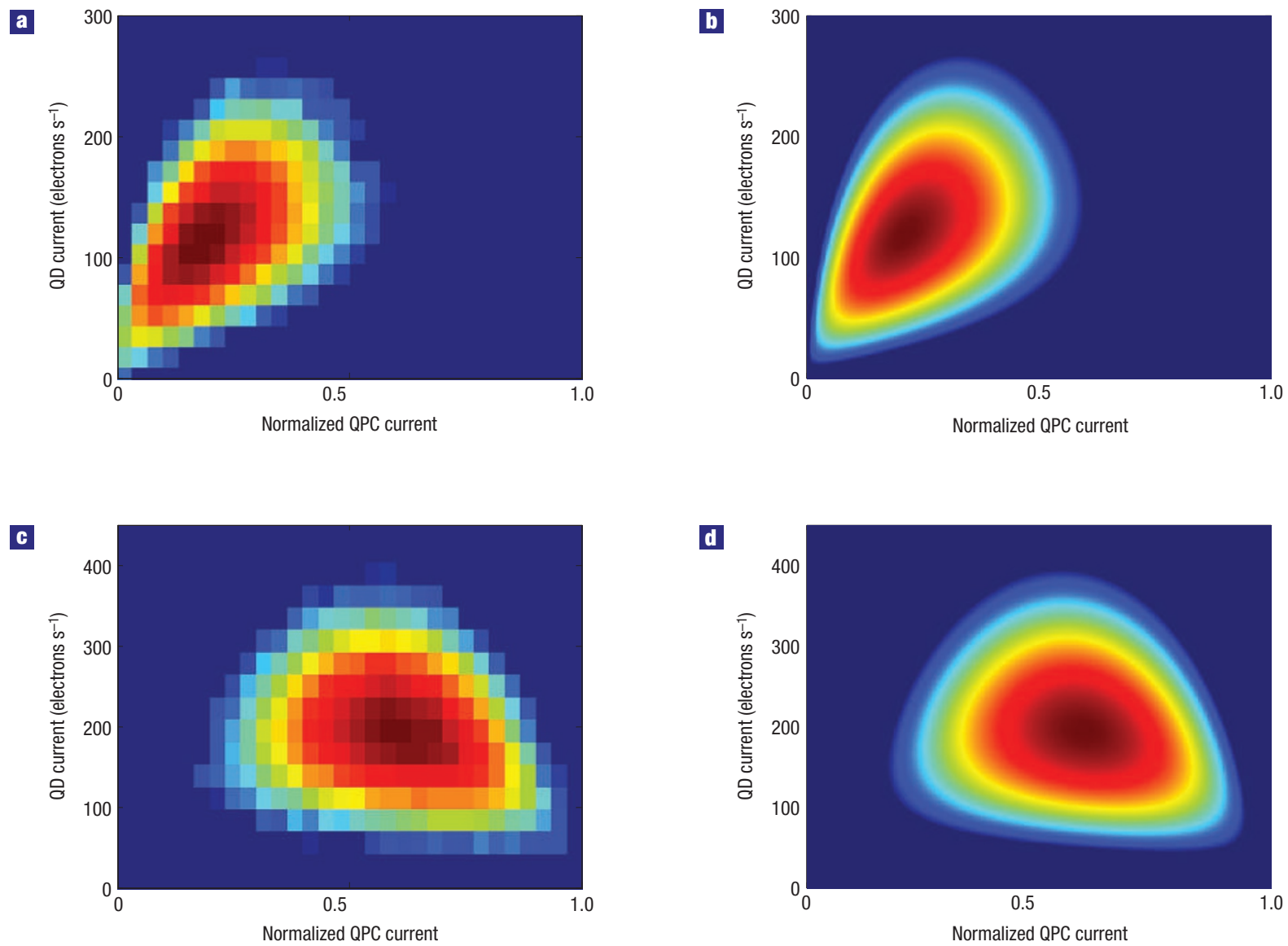

Figure 2 Joint probability distributions. The logarithm of the joint probability distribution of detecting QPC current ( $x$ axis) and QD current ( $y$ axis) is given as a colour-density plot, where red indicates high probability and blue indicates low probability. a,c, Experimental constructions for data sets A and B respectively. The experimental probability distributions were generated by splitting the data into a large number of subtraces, each containing on average seven tunnelling events. b,d, Theoretical predictions for configurations A and B respectively; see the Supplementary Information for an explicit formula.

generalizing previous results ${ }^{27,28}$. Results (1) and (2) follow from (3). The function $H(0, \chi)$ generates the current cumulants of the $\mathrm{QD}^{27}$ (the first few of which have been experimentally verified $^{22,29}$ ), and $H(\lambda, 0)$ generates the current cumulants of the $\mathrm{QPC}^{28}$. The full QPC current distribution was predicted to have an elliptical shape ${ }^{28}: \log P(I) / t=-\left(G_{1}-G_{2}\right)^{2} /(2 \Delta I)$, where $G_{1,2}=\sqrt{\Gamma_{1,2}\left|I-I_{2,1}\right|}$. This prediction is experimentally confirmed in Fig. $1 \mathrm{~b}$ for data sets $\mathrm{A}$ (blue asterisks) and B (red squares) with no fitting (see the Methods section). The joint generating function (3) is directly related to the joint probability distribution of measuring current $I$ and current $J$ (see Supplementary Information, equation (6)). The logarithm of this distribution has been measured and given in Fig. 2a,c for configurations A and B respectively. The theoretical prediction for this quantity is given in Fig. $2 b, \mathrm{~d}$ with striking agreement.

Having described the joint statistical properties of both currents, we now return to the detection question. It is important to distinguish between physical back-action and statistical-informational back-action. The non-invasive QPC detector changes its physical current state depending on whether or not the QD is occupied by an extra electron, whereas the physical dynamics of the QD is unaffected by the state of the QPC. However, by observing a particular outcome of the detector variable $(I)$, this leads to conditional (Bayesian) back-action of the detector on the system variable $(J)$. This informational back-action, or constrained randomness, manifests itself in a variety of novel effects. We introduce the concept of conditional counting statistics: the statistical current fluctuations of one system, given the observation of a given current in the other. These statistics may be calculated from the joint generating function (3) (see the Supplementary Information), yielding a mixed generator $H_{1}(I, \chi)$ of the (normalized) conditional statistics of the QD, given the observation of a current $I$,

$$
H_{1}=\Omega\left(\mathrm{e}^{\chi / 2}-1\right), \quad \Omega=\frac{\sqrt{\Gamma_{1} \Gamma_{2}}}{\Delta I} \sqrt{\left(I-I_{1}\right)\left(I_{2}-I\right)} .
$$

Taking derivatives, all conditional cumulants are given by $\left\langle\left\langle J^{n}\right\rangle_{c}=\Omega / 2^{n}\right.$, yielding a set of universal semicircles as a function of the current $I$. At the endpoints of the interval, the QPC current is observed to remain in $I_{1}$ or $I_{2}$, and therefore there cannot be any QD current, or any associated noise. The conditional QD current cumulants all have a maximum at $I_{0}$. The conditional current maximum $\sqrt{\Gamma_{1} \Gamma_{2}} / 2$ is always larger than the unconditional current $\langle J\rangle$. Figure $3 \mathrm{a}, \mathrm{b}$ compares the experimental values of the first two conditional cumulants to the theoretical semicircles, with excellent agreement. The distribution described by the generating function (4) is a poissonian distribution with a generalized rate 

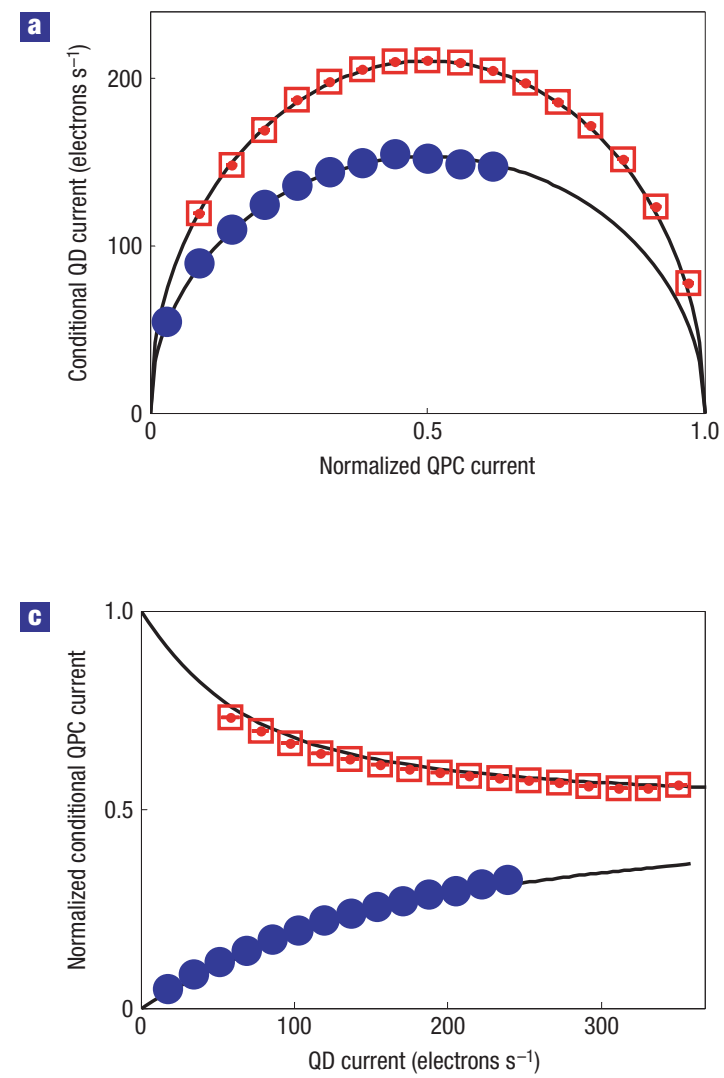
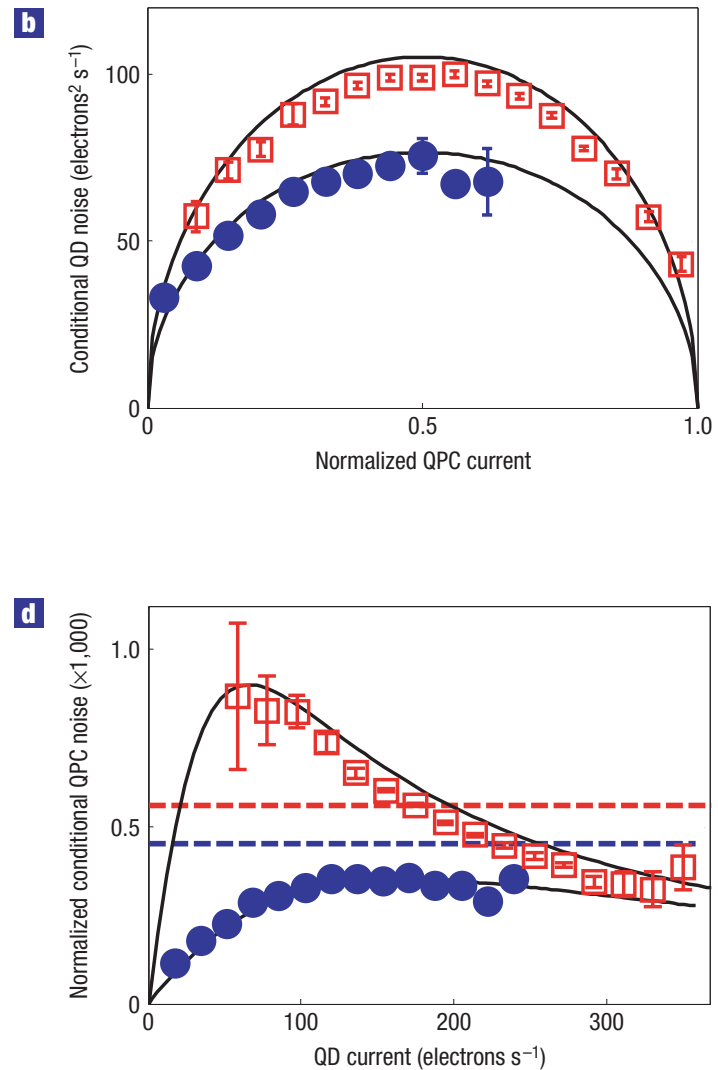

Figure 3 Conditional current cumulants. a, Conditional QD current $\langle J\rangle_{c}$ plotted as a function of $I . \mathbf{b}$, Conditional QD noise $\left\langle\left\langle J^{2}\right\rangle_{c}\right.$ plotted as a function of $I$. The solid lines in $\mathbf{a}$ and $\mathbf{b}$ are the theory from equation (4), describing universal semicircles. Filled blue circles and open red squares denote data sets A and B respectively. c, Conditional QPC current $\langle I\rangle_{\mathrm{c}}$ plotted as a function of $J$. d , Conditional QPC noise $\left\langle I^{2}\right\rangle_{\mathrm{c}}$ plotted as a function of $J$. The solid lines in $\mathbf{c}$ and $\mathbf{d}$ are the theory, given by equation (5). In $\mathbf{d}$ the horizontal dashed lines indicate the unconditional noise level, illustrating the effect of 'conditional noise enhancement' for data set B described in the text. The experimental statistics were generated by splitting the data into a large number of subtraces, each containing on average four tunnelling events. The error bars show the standard error, evaluated by dividing all the data into 10 subsets and calculating the moments for each subset individually.

$\Omega$, and effective charge $e^{*}=e / 2$, showing a radical change when compared with the unconditional distribution ${ }^{27}$.

Turning our perspective around, we can pose similar questions about the conditional detector statistics, given an observation of the system current $J$. Although the generating function $H_{2}(\lambda, J)$ for these statistics may be found analytically (see the Supplementary Information), we focus on the conditional current $\langle I\rangle_{c}$, and the conditional noise $\left\langle\left\langle I^{2}\right\rangle_{c}\right.$, given by

$$
\langle I\rangle_{\mathrm{c}}=I_{0}-\frac{\Delta I \Delta \Gamma}{J+S}, \quad\left\langle\left\langle I^{2}\right\rangle_{\mathrm{c}}=\frac{(\Delta I)^{2} J}{S(J+S)},\right.
$$

where $S=\sqrt{J^{2}+(\Delta \Gamma)^{2}}$. These conditional cumulants are experimentally calculated and compared with equation (5) in Fig. 3c,d. As a function of the QD current $J$, the conditional current tends to either $I_{1}$ or $I_{2}$ as $J \rightarrow 0$, depending on the sign of $\Delta \Gamma$. This corresponds to the most likely detector current configuration in the event of no switches being observed: the QPC current stays at one value, also implying that the system becomes noiseless in this limit. This is easily seen in (5) because $\left\langle\left\langle I^{2}\right\rangle_{c}\right.$ is proportional to $J$. The exception to this rule is the perfectly symmetric situation $\Gamma_{1}=\Gamma_{2}$, where the QPC conditional average current is $I_{0}$. This situation corresponds to rare symmetric switching between the states, whose effective rate is the conditional QD current $J$. The corresponding QPC conditional noise actually diverges in this limit, because the effective switching rate is vanishing. This effect, where the noise in one system (monitored by another) can be dramatically larger than the unmonitored noise, we refer to as conditional noise enhancement. The same effect persists in the asymmetric situation, and the maximum of the conditional noise occurs at $J^{2}=(\Delta \Gamma)^{2}(\sqrt{5}-1) / 2$. For the conditional noise peak to exceed the unconditional noise, the ratio $R=|\Delta \Gamma| \Gamma_{1} \Gamma_{2} / \Gamma_{0}^{3}$ must be less than $[(\sqrt{5}-1) / 2]^{5 / 2} \approx 0.3$. For data sets $\mathrm{A}$ and $\mathrm{B}$, $R_{\mathrm{A}} \approx 0.38$ and $R_{\mathrm{B}} \approx 0.19$, so only data set $\mathrm{B}$ shows conditional noise enhancement. In the opposite limit, $J \rightarrow \infty$, the conditional current tends to $I_{0}$, and the noise tends to zero. This situation corresponds to rapid symmetric switching between the current states, whose effective rate is again controlled by $J$. In both limits, the typical dynamics of the telegraph process is completely taken over by the transport condition.

\section{METHODS}

We now discuss the experimental procedure. From a measurement such as shown in Fig. 1c, we can directly determine the rates $\Gamma_{1,2}$ for electrons tunnelling into and out of the $\mathrm{QD}^{6,30}$. The tunnelling rates are controlled by tuning the voltages applied to gates G1 and G2. The data presented here were taken at two different gate voltage configurations. Configuration $\mathrm{A}$ is characterized by $\Gamma_{1}=160 \mathrm{~Hz}, \Gamma_{2}=586 \mathrm{~Hz}$, and configuration B is characterized by $\Gamma_{1}=512 \mathrm{~Hz}, \Gamma_{2}=345 \mathrm{~Hz}$. For each configuration, we collected traces of length $T=700 \mathrm{~s}$, containing around $10^{5}$ tunnelling events. 


\section{SAMPLE FABRICATION}

Figure la shows the sample used in the experiments reported here. The structure was fabricated using scanning probe lithography on a

GaAs $/ \mathrm{Al}_{0.3} \mathrm{Ga}_{0.7} \mathrm{As}$ heterostructure containing a two-dimensional electron gas $34 \mathrm{~nm}$ below the surface.

\section{SAMPLE CHARACTERISTICS}

The measurements were carried out in a ${ }^{3} \mathrm{He} /{ }^{4} \mathrm{He}$ dilution refrigerator with an electron temperature of about $190 \mathrm{mK}$, as determined from the width of thermally broadened Coulomb blockade resonances. The charging energy of the QD is $2.1 \mathrm{meV}$ and the mean level spacing is $200-300 \mu \mathrm{eV}$.

\section{EXPERIMENTAL METHOD}

The conductance of the QPC, $G_{\mathrm{QPC}}$, was tuned close to $0.5 \times 2 e^{2} / h$. We apply a d.c. bias voltage between the source and the drain of the QPC, $V_{\mathrm{QPC}}=250 \mu \mathrm{V}$, and measure the current through the QPC, $I_{\mathrm{QPC}}$, which depends on the number of electrons $N$ in the QD. The current signal was digitized with a sampling frequency of $100 \mathrm{kHz}$, thereafter software filtered at $4 \mathrm{kHz}$ using an eighth-order Butterworth filter. To avoid tunnelling due to thermal fluctuations, the QD was voltage biased with a bias much larger than the temperature.

Received 3 November 2006; accepted 24 January 2007; published 11 March 2007.

References

1. Lu, W. et al. Real-time detection of electron tunnelling in a quantum dot. Nature $\mathbf{4 2 3}$, $422-425$ (2003)

2. Fujisawa, T. et al. Electron counting of single-electron tunneling current. Appl. Phys. Lett. 84, 2343-2345 (2004)

3. Bylander, J., Duty, T. \& Delsing, P. Current measurement by real-time counting of single electrons. Nature 434, 361-364 (2005).

4. Field, M. et al. Measurements of Coulomb blockade with a noninvasive voltage probe. Phys. Rev. Lett. 70, 1311-1314 (1993).

5. Elzerman, J. M. et al. Single-shot read-out of an individual electron spin in a quantum dot. Nature 430, 431-435 (2004).

6. Schleser, R. et al. Time-resolved detection of individual electrons in a quantum dot. Appl. Phys. Lett. 85, 2005-2007 (2004).

7. Vandersypen, L. M. K. et al. Real-time detection of single-electron tunneling using a quantum point contact. Appl. Phys. Lett. 85, 4394-4396 (2004).

8. Sprinzak, D., Buks, E., Heiblum, M. \& Shtrikman, H. Controlled dephasing of electrons via a phase sensitive detector. Phys. Rev. Lett. 84, 5820-5823 (2000).

9. Blanter, Y. M. \& Büttiker, M. Shot noise in mesoscopic conductors. Phys. Rep. 336, 1-166 (2000)

10. Machlup, S. Noise in semiconductors: Spectrum of a two-parameter random signal. J. Appl. Phys. 25 341-343 (1954).
11. Dresselhaus, P. D., Ji, L., Han, S., Lukens, J. E. \& Likharev, K. K. Measurement of single electron lifetimes in a multijunction trap. Phys. Rev. Lett. 72, 3226-3229 (1994).

12. Kogan, S. Random telegraph noise in microstructures. Phys. Rev. Lett. 81, 2986-2989 (1998).

13. Wacker, A. \& Schöll, E. Criteria for stability in bistable electrical devices with S- or Z-shaped current voltage characteristic. J. Appl. Phys. 78, 7352-7357 (1995).

14. Landauer, R. Fluctuations in bistable tunnel diode circuits. J. Appl. Phys. 33, 2209-2216 (1962).

15. Hänggi, P. \& Thomas, H. Stochastic processes: Time-evolution, symmetries and linear response. Phys. Rep. 88, 207-319 (1982).

16. Blanter, Y. M. \& Büttiker, M. Transition from sub-poissonian to super-poissonian shot noise in resonant quantum wells. Phys. Rev. B 59, 10217-10226 (1999).

17. Sukhorukov, E. V., Burkard, G. \& Loss, D. Noise of a quantum dot system in the cotunneling regime. Phys. Rev. B 63, 125315 (2001).

18. Wernsdorfer, W. et al. Experimental evidence of the Neel-Brown model of magnetization reversal. Phys. Rev. Lett. 78, 1791-1794 (1997).

19. Ingvarsson, S. et al. Low-frequency magnetic noise in micron-scale magnetic tunnel junctions. Phys. Rev. Lett. 85, 3289-3292 (2000).

20. Urazhdin, S., Birge, N. O., Pratt, W. P. \& Bass, J. Current-driven magnetic excitations in permalloy-based multilayer nanopillars. Phys. Rev. Lett. 91, 146803 (2003).

21. Bezrukov, S. M. \& Kasianowicz, J. J. Current noise reveals protonation kinetics and number of ionizable sites in an open protein ion channel. Phys. Rev. Lett. 70, 2352-2355 (1993).

22. Gustavsson, S. et al. Counting statistics of single electron transport in a quantum dot. Phys. Rev. Lett. 96, 076605 (2006).

23. Fujisawa, T., Hayashi, T., Tomita, R. \& Hirayama, Y. Bidirectional counting of single electrons. Science 312, 1634-1636 (2006).

24. Fuhrer, A. et al. Electronic properties of nanostructures defined in $\mathrm{Ga}[\mathrm{Al}] \mathrm{As}$ heterostructures by local oxidation. Superlatt. Microstruct. 31, 19-42 (2002).

25. Levitov, L. S., Lee, H. \& Lesovik, G. B. Electron counting statistics and coherent states of electric current. J. Math. Phys. 37, 4845-4866 (1996).

26. Korotkov, A. N. Continuous quantum measurement of a double dot. Phys. Rev. B 60 5737-5742 (1999).

27. Bagrets, D. A. \& Nazarov, Yu. V. Full counting statistics of charge transfer in coulomb blockade systems. Phys. Rev. B 67, 085316 (2003).

28. Jordan, A. N. \& Sukhorukov, E. V. Transport statistics of bistable systems. Phys. Rev. Lett. 93 , 260604 (2004).

29. Gustavsson, S. et al. Measurements of higher-order noise correlations in a quantum dot with a finite bandwidth detector. Phys. Rev. B 75, 075314 (2007).

30. Naaman, O. \& Aumentado, J. Poisson transition rates from time-domain measurements with a finite bandwidth. Phys. Rev. Lett. 96, 100201 (2006).

\section{Acknowledgements}

We acknowledge the support of the Swiss National Science Foundation. We thank M. Reinwald and W. Wegscheider at the Institut für experimentelle und angewandte Physik Universität Regensburg, Germany, for fabricating the wafers used in this experiment.

Correspondence and requests for materials should be addressed to E.V.S.

Supplementary Information accompanies this paper on www.nature.com/naturephysics.

Competing financial interests

The authors declare no competing financial interests.

Reprints and permission information is available online at http://npg.nature.com/reprintsandpermissions/ 